\title{
Synthesis and Application of Blocked Waterborne Polyurethane Crosslinker
}

\author{
Xingyuan Ma ${ }^{1, a}$, Xiaohan Qiu ${ }^{1, b}$, Shufang Zhang ${ }^{1, \mathrm{c}}$ \\ ${ }^{1}$ College of Resource \& Environment, Shaanxi University of Science and Technology, Xi'an, 710021, \\ China \\ aemail: maleather@163.com, bemail: qiuxiaohanpu@163.com, cemail: jiazsf@163.com
}

Keywords: Blocked Polyurethane; Waterborne Polyurethane; Crosslinker; Synthesis; Application

\begin{abstract}
In this paper, the blocked waterborne polyurethane crosslinker were prepared by polyaddition reaction with isophorone diisocyanate (IPDI), polypropylene glycol-2000 (PPG MW2000) and dimethylolpropionic acid (DMPA) as internal emulsifier, sodium bisulfite $\left(\mathrm{NaHSO}_{3}\right)$ as blocked agent in presence of dibutyltin dilaurate (DBTDL) as catalyst. The Fourier transform infrared spectrum analysis (FT-IR) showed that the isocyanate groups of blocked waterborne polyurethane crosslinker are completely blocked. The differential scanning calorimetry (DSC) analysis showed that the deblocking temperature of blocked waterborne polyurethane crosslinker is between 88.3C 92.4C. Application results showed that the blocked waterborne polyurethane crosslinker can significantly improve the tensile strength and water resistance of waterborne polyurethane membrane.
\end{abstract}

\section{Introduction}

Polyurethane is a versatile polymer material, with a wide variety of physical and chemical properties that can be obtained from many commercially available and relatively cheap polyols, isocyanates and chain extender [1]. Waterborne polyurethane dispersions can solve the pollution problem of solvent-based polyurethane. But waterborne polyurethane present drawbacks such as poor surface properties, poor solvent resistance and water resistance, limited mechanical properties caused principally by low crosslinking density. Therefore, the best way to improve performance of waterborne polyurethane is to increase the degree of crosslinking [2].

However, high level of crosslinking can't be introduced into waterborne polyurethane easily due to the problems of high viscosity and poor emulsification of the prepolymers. Crosslinkers currently in use display certain human and/or environmental toxicity characteristics. As examples, melamine formaldehyde and aziridine, both commonly used agents, release harmful vapors and can produce adverse reactions.

Blocked waterborne polyurethane crosslinker which the end isocyanate groups are blocked [3] [4]. The blocked isocyanate groups could be released at de-blocking temperature, which could react with active hydrogen in waterborne polyurethane and produce three-dimensional crosslinked structure. Therefore, the blocked polyurethane is a good crosslinkier of waterborne polyurethane which can significantly improve the tensile strength and water resistance [5] [6] [7].

\section{Experimental}

Materials and instruments. Polypropylene glycol (PPG; MW2000) (supplied by Guangdong Dongguang chemical reagent company), isophorone diisocyanate (IPDI; provided by Evonik industries AG), dimethylol propionic acid (DMPA; supplied by Aladdin chemical reagent company). Dibutyltin dilaurate (DBTDL; provided by Xi'an chemical reagent factory), triethylamine (TEA; supplied by Tianjin Hongyan chemical reagent factory), N-methyl-2-pyrrolidone (NMP; provided by Tianjin Yongsheng fine chemical Co., Ltd.), sodium bisulfite (NaHSO3; supplied by Tianjin Yongsheng fine chemical Co., Ltd.), waterborne polyurethane (DG-6915; solid content is 30\%, provided by Yantai Huada chemical industry Co., Ltd.). Fourier transform infrared spectrometer 
(Perkin-Elmer specturum 22000; provided by American PERKIN ELMER Company). Differential scanning calorimeter (DSC200PC; provided by Germany NETZSCH company). Electronic tensile machine (LDW-1; provided by Jinan Eastern test Instrument Co.,Ltd.)

Preparation process of blocked waterborne polyurethane crosslinker. The schematic diagram of synthesis is showed in Fig.1.

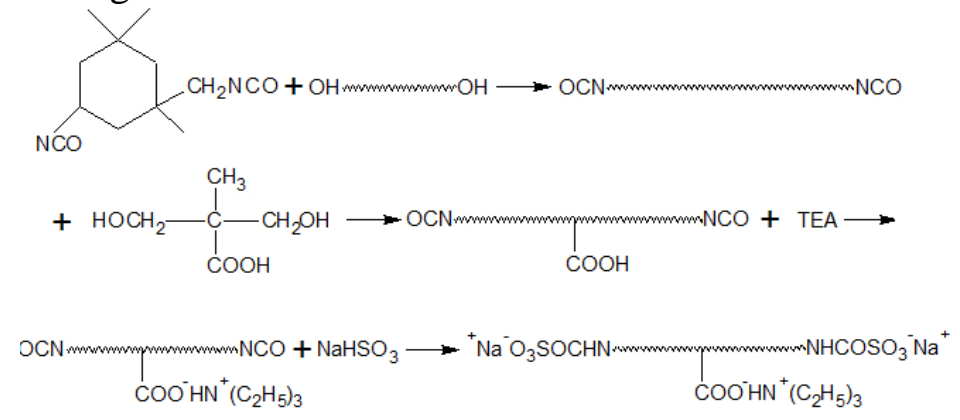

Fig. 1 The schematic diagram of synthesis of enclosed waterborne polyurethane crosslinker

PPG-2000 and IPDI were charged into the flask and mixture was heated at the prepolymerization temperature in the presence of DBTDL, the reaction was carried out until the theoretical NCO values. Afterwards, calculated amount of DMPA dissolved in NMP was added into the flask, and the reaction was carried out the theoretical NCO values were obtained. Then, the reaction mixture was cold to 30C, and then TEA was added to neutralize the carboxylic acids groups in the DMPA during the next 30 minutes. The reaction mixture was cold to $10 \mathrm{C}$ and then an aqueous solution of $\mathrm{NaHSO}_{3}$ was added into the flask, constant stirring and the reaction was carried out until the theoretical NCO values were obtained. Finally, calculated amount of deionized water was added into the flask under vigorous stirring. After emulsification, the blocked waterborne polyurethane crosslinker could be obtained.

The influence of $\mathbf{R}$-value on the blocked waterborne polyurethane crosslinker. A series of blocked waterborne polyurethane were prepared under conditions of the different R-value, the appearance and stability of the emulsion was observed, and the blocking rate of NCO groups (determined by the iodimetry method) was measured.

The influence of DMPA on the blocked waterborne polyurethane crosslinker. A series of blocked waterborne polyurethane were prepared under conditions of the different amount of DMPA, the appearance and stability of the emulsion was observed, and the blocking ratio of NCO groups was measured.

Preparation of waterborne polyurethane films. For some experiments, solid polyurethane films were used under conditions of the different mixing solid content ratio (the blocked waterborne polyurethane crosslinker: DG-6915). They were prepared by casting emulsion in a PTFE mould, followed by drying at room temperature for $24 \mathrm{~h}$. The resulting films were then heated in an oven at $100 \mathrm{C}$ for $2 \mathrm{~h}$ to ensure the complete removal water in the film.

\section{Measurements}

Tensile strength. The method of determination based on QB/T 2710-2005, tensile strength and elongation at the break of the films were measured on a electronic tensile machine LDW-1. Three parallel measurements were carried out and averaged.

Stability of the emulsion. The blocked waterborne polyurethane crosslinker emulsion was allowed to store 5 months, observe and record the sedimentation and stratification of emulsion.

Water resistance. The water resistance was determined as follows. The films were cut into 25 $\mathrm{mm} \times 25 \mathrm{~mm}$ and dried in an oven at 40C to determine their dry weight $\left(\mathrm{W}_{1}\right)$. Then the films were immersed in distilled water at room temperature for 24h, followed by wiping off the surface water with a piece of filter paper to determine their weight immediately $\left(\mathrm{W}_{2}\right)$. The water absorption (W\%) was calculated by the formula: $W(\%)=\frac{W_{2}-W_{1}}{W_{1}} \times 100$. 


\section{Results and discussion}

The influence of R-value (-NCO/-OH) on the emulsion. Experimental results are shown in Tab.1.

Tab.1 The influence of $\mathrm{R}$ value on the appearance and stability of the blocked polyurethane

\begin{tabular}{ccccccc}
\hline R-value & 1.2 & 1.4 & 1.6 & 1.8 & 2.1 & 2.5 \\
\hline $\begin{array}{c}\text { Appearance } \\
\text { of emulsion }\end{array}$ & $\begin{array}{c}\text { Transparent, } \\
\text { Pan-blue } \\
\text { light }\end{array}$ & $\begin{array}{c}\text { Transparent, } \\
\text { Pan-blue } \\
\text { light }\end{array}$ & $\begin{array}{c}\text { Translucent, } \\
\text { Pan-blue } \\
\text { light }\end{array}$ & $\begin{array}{c}\text { Translucent, } \\
\text { pale yellow }\end{array}$ & $\begin{array}{c}\text { Translucent, } \\
\text { pale yellow }\end{array}$ & $\begin{array}{c}\text { Opaque, } \\
\text { pale yellow }\end{array}$ \\
\hline $\begin{array}{c}\text { Stability of } \\
\text { emulsion }\end{array}$ & $\begin{array}{c}\text { Uniform and } \\
\text { stable }\end{array}$ & $\begin{array}{c}\text { Uniform and } \\
\text { stable }\end{array}$ & $\begin{array}{c}\text { Uniform and } \\
\text { stable }\end{array}$ & $\begin{array}{c}\text { Uniform and } \\
\text { stable }\end{array}$ & $\begin{array}{c}\text { Slight } \\
\text { stratified }\end{array}$ & $\begin{array}{c}\text { Fully } \\
\text { stratified }\end{array}$ \\
\hline
\end{tabular}

Tab.1 shows that the transparency and stability of emulsion was reduced when $\mathrm{R}$ value of the prepolymerization increased. The reason is that the free NCO groups increase in the reaction system as the R value increases, and more NCO groups react with water to produce a large amount of urea bond, resulting in viscosity of system increased, the particle size of emulsion increased, the appearance of emulsion changes from transparent to opaque, stability of emulsion becomes deteriorated.

The influence of R-value on blocked reaction. Experimental result is showed in Fig.2.

Fig.2 shows that the blocked ratio increased with the increase of the R-value when 1.6> $\mathrm{R}$-value $>1$, the blocked ratio decreased with the increase of the $\mathrm{R}$-value when $\mathrm{R}$-value $>1.6$. The reason is that the free NCO groups increase in the reaction system as the R-value increases. Within a certain range, the increase of R-value resulted in the decrease of viscosity of the system. It is benefical to the blocking reaction, so the blocked ratio increases. When the R-value increases more than 1.6, excessive NCO groups react with water to produce a large amount of urea bond, resulting in the hydrophilic of system worse. So the blocked ratio decreases. Experimental results showed that the optimum of R-value of prepolymerization is 1.6.

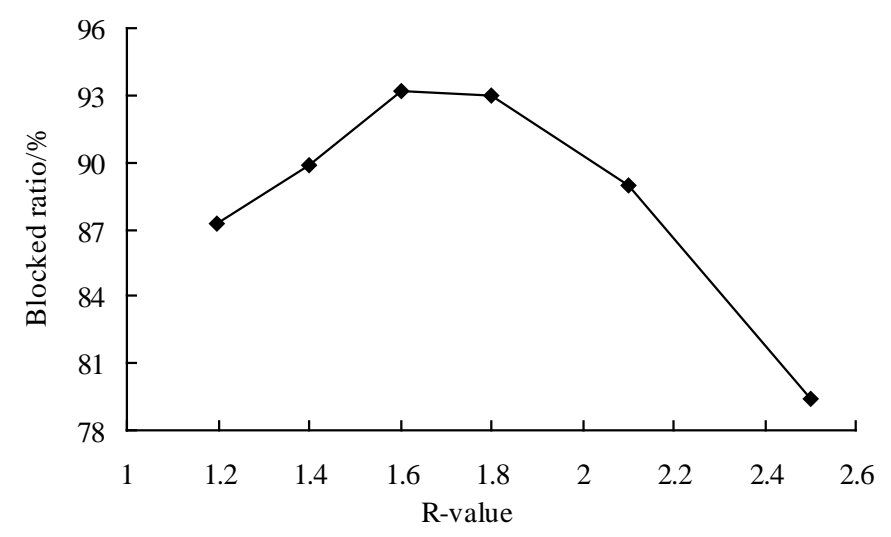

Fig.2 The relation curve between R-value and blocked ratio

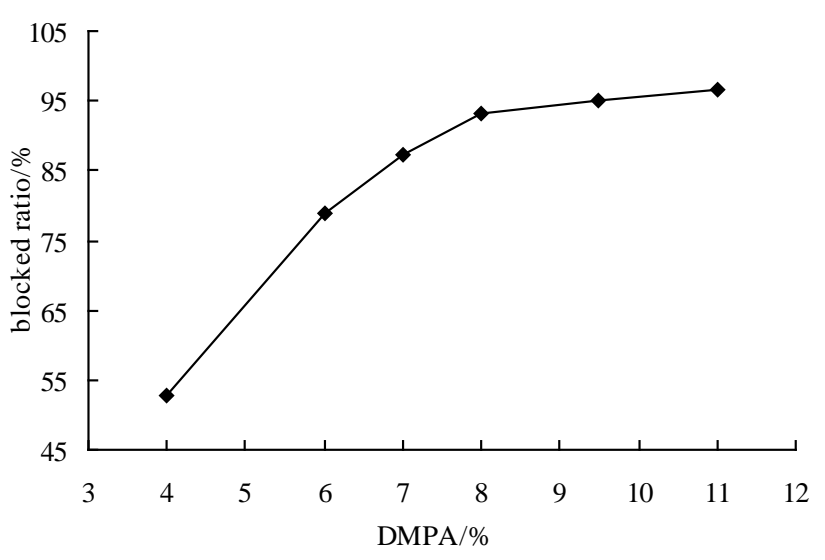

Fig.3 The relation curve between DMPA/\% and blocked ratio

The influence of DMPA on blocked reaction. Experimental results are shown in Fig.3.

Fig.3 shows that the blocked ratio increased as the amount of DMPA increased. When the amount of DMPA reached 8\%, blocked ratio could reach above $90 \%$. The amount of DMPA continued to increase, the increase of blocked ratio is not obvious. The reason is that the polyurethane prepolymer hydrophobic substance is an oil phase and the aqueous solution of sodium bisulfite is blocking agent, so homogeneous reaction is a problem. In this experiment, the polyurethane prepolymer had hydrophilicity to achieve homogeneous reaction by introducing hydrophilic group to the polyurethane. So that the hydrophilicity of prepolymer increased when the amount of DMPA increased, and the blocked ratio increased. However, when the amount of DMPA is too high, application performance and water resistance of the blocked waterborne polyurethane crosslinker are deteriorated. In summary, the optimum amount of DMPA is 8\%.

The influence of DMPA on the stability of the emulsion. Experimental result is showed in Tab.2. 
Tab.2 The influence of DMPA on the appearance and stability of the blocked polyurethane

\begin{tabular}{ccccccc}
\hline DMPA/\% & 4 & 6 & 7 & 8 & 9.5 & 11 \\
\hline $\begin{array}{c}\text { Appearance } \\
\text { of emulsion }\end{array}$ & $\begin{array}{c}\text { Opaque, } \\
\text { Milky white }\end{array}$ & $\begin{array}{c}\text { Translucent, } \\
\text { pale yellow }\end{array}$ & $\begin{array}{c}\text { Translucent, } \\
\text { pale yellow }\end{array}$ & $\begin{array}{c}\text { Translucent, } \\
\text { Pan-blue } \\
\text { light }\end{array}$ & $\begin{array}{c}\text { Transparent, } \\
\text { Pan-blue } \\
\text { light }\end{array}$ & $\begin{array}{c}\text { Transparent, } \\
\text { Pan-blue } \\
\text { light }\end{array}$ \\
$\begin{array}{c}\text { Stability of } \\
\text { emulsion }\end{array}$ & $\begin{array}{c}\text { Fully } \\
\text { stratified }\end{array}$ & $\begin{array}{c}\text { Slight } \\
\text { stratified }\end{array}$ & $\begin{array}{c}\text { Uniform and } \\
\text { stable }\end{array}$ & $\begin{array}{c}\text { Uniform and } \\
\text { stable }\end{array}$ & $\begin{array}{c}\text { Uniform and } \\
\text { stable }\end{array}$ & $\begin{array}{c}\text { Uniform and } \\
\text { stable }\end{array}$ \\
\hline
\end{tabular}

Tab.2 shows that the transparency and stability of emulsion was increased when the amount of DMPA increased. The reason is that the hydrophilicity of emulsion increased when the amount of DMPA increased. During the emulsification process, the hydrophilicity of the system increases, emulsification process is easier, the particle size is smaller, the emulsion stability is better.

The application performance of blocked waterborne polyurethane crosslinker. Experimental result is showed in Tab.3.

Tab.3 The influence of amount of crosslinkers on tensile strength of polyurethane membrane

\begin{tabular}{cccc}
\hline $\begin{array}{c}\text { The crosslinker } \\
\text { /\% }\end{array}$ & $\begin{array}{c}\text { The tensile strength } \\
/\left(\mathrm{N} \cdot \mathrm{mm}^{-2}\right)\end{array}$ & $\begin{array}{c}\text { The elongation at break } \\
/ \%\end{array}$ & $\begin{array}{c}\text { The ratio of water } \\
\text { absorption/\% }\end{array}$ \\
\hline 0 & 8.93 & 408 & 21.80 \\
5 & 12.33 & 512 & 17.19 \\
10 & 16.00 & 628 & 15.23 \\
15 & 14.25 & 522 & 28.46 \\
20 & 8.45 & 345 & 35.38 \\
\hline
\end{tabular}

Tab.3 shows that adding appropriate amount of blocked waterborne polyurethane crosslinker can significantly improve the tensile strength and elongation at break of waterborne polyurethane membrane, when the addition amount of waterborne polyurethane crosslinker is $10 \%$, the tensile strength of waterborne polyurethane membrane is highest. The reason is that adding appropriate amount of waterborne polyurethane crosslinker, the deblocked isocyanate groups increase, the degree of crosslinking of waterborne polyurethane membrane increases, and thus the tensile strength of the waterborne polyurethane membrane increases. However, when the addition amount of waterborne polyurethane crosslinker is too much, the tensile strength of waterborne polyurethane membrane decreases due to excessive crosslinking. On the other hand, the deblocking reaction will produce more inorganic salt which filled in the waterborne polyurethane membrane that reduces water resistance and tensile strength of waterborne polyurethane membrane. Experimental results showed that the optimum addition amount of waterborne polyurethane crosslinker is $10 \%$.

FT-IR analysis. The result is showed in Fig.4.

Fig. 4 shows that -NCO absorption peak disappeared at $2262 \mathrm{~cm}^{-1}$, indicating the -NCO has been completely blocked; the absorption peak at $3423 \mathrm{~cm}^{-1}$ originates from $\mathrm{N}-\mathrm{H}$ stretching vibration, $\mathrm{C}=\mathrm{O}$ stretching vibration peak is at $1656 \mathrm{~cm}^{-1}$, the absorption peak at $1520 \sim 1560 \mathrm{~cm}^{-1}$ originates from N-H deformation vibration, indicating the carbamate was generated. There are characteristic peaks of $-\mathrm{CH}_{3}$ and $-\mathrm{CH}_{2}\left(2700 \sim 3000 \mathrm{~cm}^{-1}\right)$, C-O $\left(1225 \sim 1235 \mathrm{~cm}^{-1}\right)$ and C-O-C $\left(1060 \sim 1150 \mathrm{~cm}^{-1}\right)$ in the spectrum of polyurethane. From these analyses, this study synthesized target product.

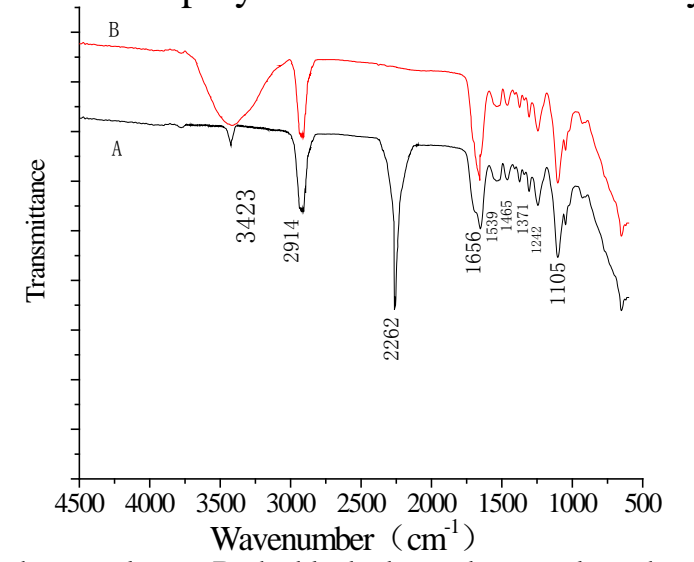

A: the prepolymer; B: the blocked waterborne polyurethane crosslinker Fig.4 FT-IR spectrograms of the prepolymer and the enclosed waterbone polyurethane crosslinke

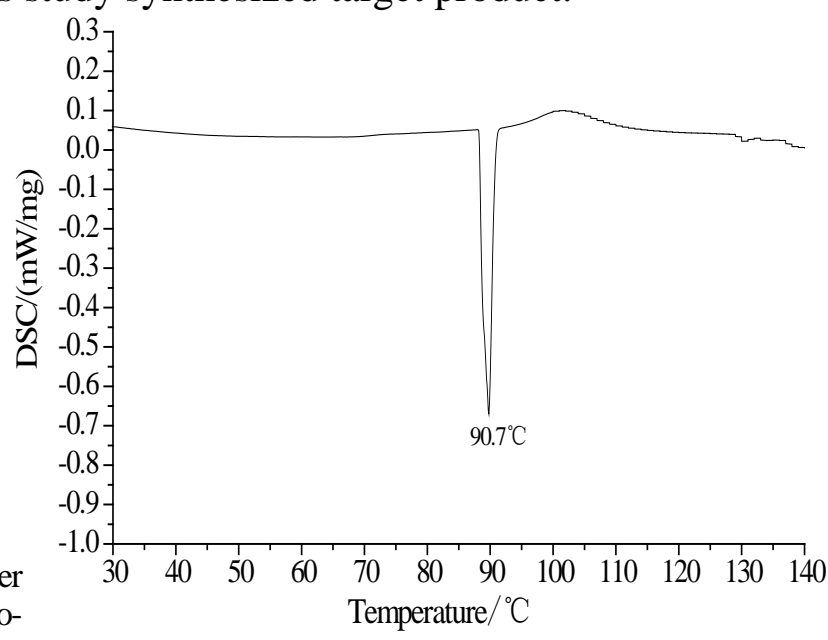

Fig.5 DSC curves of blocked waterborne polyurethane crosslinker 
DSC analysis. The result is showed in Fig.5.

Fig. 5 shows the beginning of endothermic peak at 88.3C, maximum endothermic peak at 90.7C, the end of endothermic peak at 92.4C. It is clearly shown in figure 5 that the deblocking temperature of the blocked waterborne polyurethane crosslinker between 88.3C 92.4C.

\section{Conclusion}

In this paper, blocked waterborne polyurethane has been prepared with IPDI and PPG-2000 as the main raw material, DMPA as anionic hydrophilic chain extender agent, $\mathrm{NaHSO}_{3}$ as blocking agent. The performed reaction was that the R-value was 1.6, the amount of DMPA was $8 \%$. The results of FT-IR and DSC analysis show that the -NCO groups of product are completely blocked and the deblocking temperature of product is between 88.3C 92.4C. As crosslinker, it can significantly improve the tensile strength of waterborne polyurethane membrane.

The crosslinker compares to other crosslinker, with no pollution, non-toxic, good stability, consistent with the development of green chemistry. In the field of waterborne polyurethane will have some application value.

\section{Acknowledgement}

This work was sponsored by Fujian province local area major project (Project No. 2013H4013) and China Torch Program (Project No. 2014GH030914).

\section{References}

[1] Zhang Shengwen, Liu Ren, Jiang Jinqiang. Facile synthesis of waterborne UV-curable polyurethane/silica nanocomposites and morphology, physical properties of its nanostructured films[J]. Progress in Organic Coatings, 2011, 70: 1-8.

[2] Kalele Mulonda, Zhu Yan, Mulenga Mbombo Christophe. Preparation and characterization of waterborne polyurethane crosslinked by urea bridges [J]. International Journal of Chemistry, 2011, 3(2):88-96.

[3] Cao Fengcai, Wang Wei. Synthesis and properties of blocked aliphatic waterborne polyurethane used in textile finishing agent [J]. Polyurethane Industry, 2008, 23(5): 12-15.

[4] Douglas A Wicks, Zeno W Wicks. Multistep chemistry in thin films: the challenges of blocked isocyanates [J]. Progress in Organic Coatings, 2001, 27(43): 131-140.

[5] Douglas A Wicks, Zeno W Wicks. Blocked isocyanates

III: Part A

[J]. Progress in Organic Coating, 1999, 38(36): 148-172.

[6] Jung Min Lee, Sankaraiah Subramani, Young Soo Lee, et al. Thermal decomposition behavior of blocked diisocyanates derived from mixture of blocking agents [J]. Macromolecular Research, 2005, 13(5): 427-433.

[7] Delebecq E, Pascault J P, Boutevin B, at al. On the versatility of urethane/urea bonds: reversibility, blocked Isocyanate, and non-isocyanate polyurethane [J]. Chemical Reviews, 2013, 113: 80-118. 